\title{
THE GEOMETRY OF A CLOSED FORM
}

\author{
MARISA FERNÁNDEZ, RAÚL IBÁÑEZ \\ Departamento de Matemáticas, Universidad del Pais Vasco \\ Apartado 644, 48080 Bilbao, Spain \\ E-mail:mtpferol@lg.ehu.es, mtpibtor@lg.ehu.es \\ MANUEL DE LEÓN \\ Instituto de Matemáticas y Física Fundamental \\ Consejo Superior de Investigaciones Científicas \\ Serrano 123, 28006 Madrid, Spain \\ E-mail: mdeleon@fresno.csic.es
}

\begin{abstract}
It is proved that a closed $r$-form $\omega$ on a manifold $M$ defines a cohomology (called $\omega$-coeffective) on $M$. A general algebraic machinery is developed to extract some topological information contained in the $\omega$-coeffective cohomology. The cases of 1-forms, symplectic forms, fundamental 2-forms on almost contact manifolds, fundamental 3 -forms on $G_{2}$-manifolds and fundamental 4-forms in quaternionic manifolds are discussed.
\end{abstract}

1. Introduction. As is well-known the existence of a particular geometric structure on a manifold $M$ imposes often some topological properties on it. For instance, if $\omega$ is a symplectic form on a compact manifold $M$, then $M$ has to be even dimensional, and in addition its even Betti numbers do not vanish. Moreover, a symplectic form defines a special subcomplex of the de Rham complex $\left(\Lambda^{*}(M), d\right)$ of forms on $M$ : it consists of those forms $\alpha$ which are annihilated by $\omega$, that is, $\alpha \wedge \omega=0$. Since $\omega$ is closed, we have in fact a subcomplex of $\left(\Lambda^{*}(M), d\right)$ whose cohomology is called coeffective. This cohomology was introduced and studied by Bouché [6] and related with the truncated de Rham cohomology by the class $[\omega]$. For Kähler manifolds both cohomologies are isomorphic for $k \neq n, \operatorname{dim} M=2 n$, though in general they are different for non Kähler symplectic manifolds $[1,14]$. A Nomizu's type theorem [28] was also proved in [13] and it was shown in [15] that the coeffective cohomology of a symplectic manifold of finite type is finite, so that we have introduced the coeffective numbers of the symplectic manifold and several

1991 Mathematics Subject Classification: 53C15, 46M20.

The paper is in final form and no version of it will be published elsewhere. 
inequalities relating them to the Betti numbers. Similar results were obtained in the context of almost contact $[10,16]$ and quaternionic manifolds [17].

The above results lead us to consider the following problem. Let $\omega$ be a closed $r$-form on a manifold $M$, and define the corresponding $\omega$-coeffective cohomology. This can be done since the closedness of $\omega$ implies that the operator $L_{\omega}: \alpha \leadsto \alpha \wedge \omega$ and the exterior derivative $d$ commute, so we obtain a subcomplex $\left(\mathcal{A}_{\omega}^{*}(M), d\right)$ of the de Rham complex of $M$, where $\mathcal{A}_{\omega}^{k}(M)=\operatorname{ker}\left\{L_{\omega}: \Lambda^{k}(M) \longrightarrow \Lambda^{k+r}(M)\right\}$. The resulting cohomology $H_{\omega}^{*}(\mathcal{A}(M))$ is called the $\omega$-coeffective cohomology. The point now is to relate it to the de Rham cohomology, or, in general, to extract topological information from the existence of that closed form $\omega$. In some sense, we are studying the geometry provided by $\omega$. We will use a general technique which consists in considering the five-term exact sequences associated with the long exact sequence induced by passing to cohomology from the exact short sequence

$$
0 \longrightarrow \operatorname{ker} L \longrightarrow \Lambda^{*}(M) \longrightarrow \operatorname{Im} L \longrightarrow 0 .
$$

However, to go further, we need some particular properties of the form. In this paper we collect a list of results concerning 1-forms, symplectic forms and fundamental forms on almost contact manifolds, $G_{2}$-manifolds and quaternionic manifolds. A more complete program in the Riemannian context will require the study of the fundamental forms in other $G$-structures according to Berger's list (see [2]). Another direction in a more general context will be to consider the coeffective cohomology defined for multisymplectic forms [8].

2. Coeffective cohomology. Let $M$ be a real $m$-dimensional $C^{\infty}$ differentiable manifold, $\mathfrak{X}(M)$ the Lie algebra of $C^{\infty}$ vector fields on $M$ and $\Lambda^{k}(M)$ the space of $C^{\infty} k$-forms on $M$.

If $d$ denotes the exterior derivative on $M$, then we have the de Rham differential complex

$$
\cdots \longrightarrow \Lambda^{k-1}(M) \stackrel{d}{\longrightarrow} \Lambda^{k}(M) \stackrel{d}{\longrightarrow} \Lambda^{k+1}(M) \longrightarrow \cdots
$$

whose cohomology $H^{*}(M)$ is the de Rham cohomology of $M$.

Consider now a closed $r$-form $\omega$ on $M$, and define an operator

$$
L_{\omega}: \Lambda^{k}(M) \longrightarrow \Lambda^{k+r}(M)
$$

by

$$
L_{\omega}(\alpha)=\alpha \wedge \omega .
$$

Then the space

$$
\mathcal{A}_{\omega}^{k}(M)=\operatorname{ker}\left\{L_{\omega}: \Lambda^{k}(M) \longrightarrow \Lambda^{k+r}(M)\right\}
$$

is called the subspace of $\omega$-coeffective forms on $M$. Since $\omega$ is closed, $L_{\omega}$ and $d$ commute, which implies that

$$
\cdots \longrightarrow \mathcal{A}_{\omega}^{k-1}(M) \stackrel{d}{\longrightarrow} \mathcal{A}_{\omega}^{k}(M) \stackrel{d}{\longrightarrow} \mathcal{A}_{\omega}^{k+1}(M) \longrightarrow \cdots
$$

is a differential subcomplex of the de Rham complex. Its cohomology $H_{\omega}^{k}(\mathcal{A}(M))$ is called $\omega$-coeffective cohomology of $M$. If this cohomology is finite, we define the coeffective numbers (associated to $\omega$ ) by $c_{k}(M, \omega)=\operatorname{dim} H_{\omega}^{k}(\mathcal{A}(M))$. 
3. Exact sequences and coeffective cohomology. The aim of this section is to relate the coeffective cohomology to the de Rham cohomology by means of a long exact sequence in cohomology.

Consider the following natural short exact sequence for any degree $k$ :

$$
0 \longrightarrow \operatorname{ker} L_{\omega}=\mathcal{A}_{\omega}^{k}(M) \stackrel{i}{\longrightarrow} \Lambda^{k}(M) \stackrel{L_{\omega}}{\longrightarrow} \operatorname{Im}^{k+r} L_{\omega} \longrightarrow 0
$$

Since $L_{\omega}$ and $d$ commute, (1) becomes a short exact sequence of differential complexes:

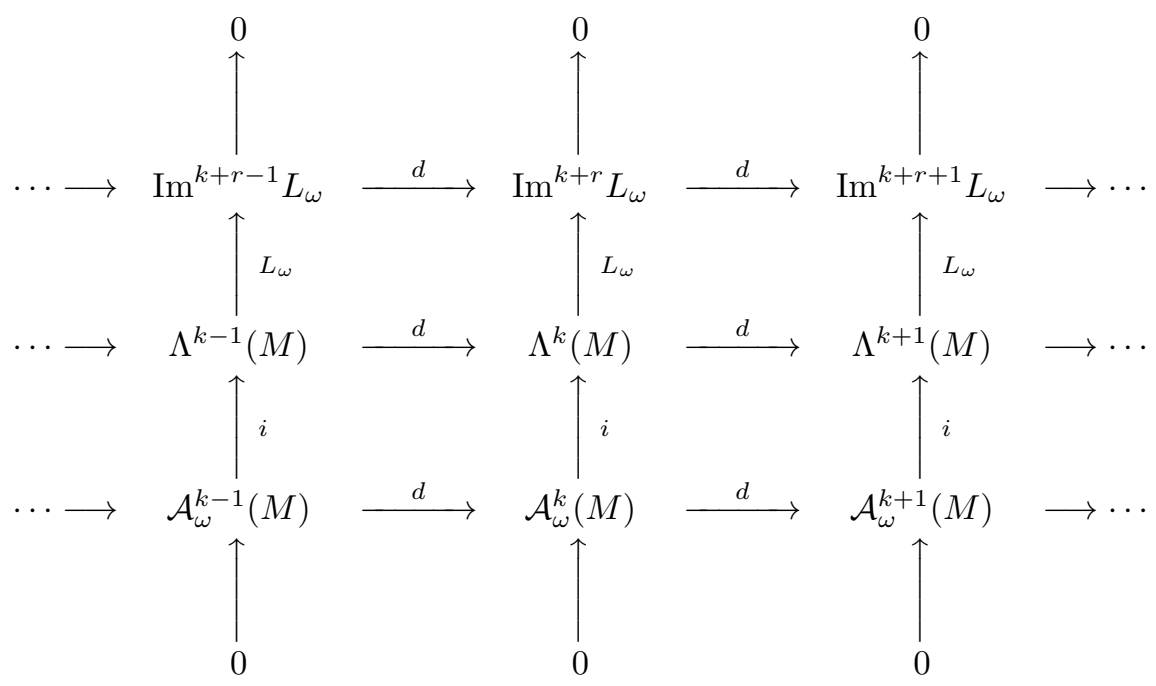

Therefore, we can consider the associated long exact sequence in cohomology:

$$
\cdots \longrightarrow H_{\omega}^{k}(\mathcal{A}(M)) \stackrel{H(i)}{\longrightarrow} H^{k}(M) \stackrel{H\left(L_{\omega}\right)}{\longrightarrow} H^{k+r}\left(\operatorname{Im} L_{\omega}\right) \stackrel{C_{k+r}}{\longrightarrow} H_{\omega}^{k+1}(\mathcal{A}(M)) \longrightarrow \cdots
$$

where $H(i)$ and $H\left(L_{\omega}\right)$ are the homomorphisms induced in cohomology by $i$ and $L_{\omega}$, respectively, and $C_{k+r}$ is the connecting homomorphism defined in the following way: if $[\alpha] \in H^{k+r}\left(\operatorname{Im} L_{\omega}\right)$, then $C_{k+r}[\alpha]=[d \beta]$, for $\beta \in \Lambda^{k}(M)$ such that $L_{\omega} \beta=\alpha$.

4. Closed 1-forms. Assume that $\omega$ is a closed 1-form without zeros. Therefore, we have

$$
H_{\omega}^{0}(\mathcal{A}(M)) \cong\{0\} .
$$

Moreover, since $\alpha \wedge \omega=0$ implies that $\alpha=\beta \wedge \omega$ we deduce that

$$
\operatorname{ker}\left\{L_{\omega}: \Lambda^{k}(M) \longrightarrow \Lambda^{k+1}(M)\right\}=\operatorname{Im}\left\{L_{\omega}: \Lambda^{k-1}(M) \longrightarrow \Lambda^{k}(M)\right\}
$$

If $M$ is of finite type, then the Betti numbers of $M, b_{k}(M)=\operatorname{dim} H^{k}(M)$ are finite. However, the coeffective cohomology groups are not finite dimensional in general. Indeed, consider the 1 -form $\omega=d x$ on $\mathbb{R}^{3}$ with standard coordinates $x, y, z$. A direct computation shows that

$$
H_{\omega}^{1}\left(\mathcal{A}\left(\mathbb{R}^{3}\right)\right) \cong C^{\infty}(\mathbb{R})
$$


Decompose the long exact sequence (2) in the following short exact sequences:

$$
0 \rightarrow \operatorname{Im} H(i)=\operatorname{ker} H\left(L_{\omega}\right) \stackrel{i}{\rightarrow} H^{k}(M) \stackrel{H\left(L_{\omega}\right)}{\rightarrow} \operatorname{Im} H\left(L_{\omega}\right) \rightarrow 0 .
$$

Then we deduce the formula:

$$
\operatorname{dim} \operatorname{ker} H\left(L_{\omega}\right)-b_{k}(M)+\operatorname{dim} \operatorname{Im} H\left(L_{\omega}\right)=0 .
$$

From (3) we obtain the following result.

Proposition 4.1. Let $M$ be a differentiable manifold with a closed 1-form $\omega$ without zeros. Assume that the $\omega$-coeffective cohomology is finite. Then we have

$$
b_{k}(M) \leq c_{k}(M, \omega)+c_{k+1}(M, \omega), \text { for all } k .
$$

Problem 4.2. What are the coeffective numbers if $\omega$ satisfies some extra geometric properties, for instance, $\omega$ is parallel with respect to a Riemannian metric?

\section{Closed 2-forms: symplectic and almost contact manifolds}

5.1. Symplectic manifolds. Let $M$ be a $2 n$-dimensional symplectic manifold with symplectic form $\omega$. From Lepage's decomposition [23] we deduce that $L_{\omega}$ is injective for $k \leq n-1$ and surjective for $k \geq n-1$. As a consequence we deduce that $\mathcal{A}_{\omega}^{k}(M)=\{0\}$, for $k \leq n-1$, and hence $H_{\omega}^{k}(\mathcal{A}(M))=\{0\}$, for $k \leq n-1$. Also, we have $H^{k+2}\left(\operatorname{Im} L_{\omega}\right)=$ $H^{k+2}(M)$, for $k \geq n$.

Now, we decompose the long exact sequence (2) in 5-term exact sequences:

$$
0 \rightarrow \operatorname{ker} H(i)=\operatorname{Im} C_{k+1} \stackrel{i}{\rightarrow} H_{\omega}^{k}(\mathcal{A}(M)) \stackrel{H(i)}{\rightarrow} H^{k}(M) \stackrel{H\left(L_{\omega}\right)}{\rightarrow} H^{k+2}(M) \stackrel{C_{k+2}}{\rightarrow} \operatorname{Im} C_{k+2} \rightarrow 0
$$

THEOREM 5.1 [15]. Let $M$ be a symplectic $2 n$-dimensional manifold of finite type. Then the coeffective cohomology group $H_{\omega}^{k}(\mathcal{A}(M))$ has finite dimension, for $k \geq n+1$. Furthermore,

$$
b_{k}(M)-b_{k+2}(M) \leq c_{k}(M, \omega) \leq b_{k}(M)+b_{k+1}(M) .
$$

REMARK 5.2. In general, the intermediate coeffective number $c_{n}(M, \omega)$ is not finite. A simple counterexample is the torus $\mathbb{T}^{2 n}=\mathbb{R}^{2 n} / \mathbb{Z}^{2 n}$ with its canonical Kähler form.

In $[15]$ we have proved the following results.

THEOREM 5.3.

(i) Let $M$ be a compact Kähler manifold of dimension $2 n$. Then we have

$$
c_{k}(M, \omega)=b_{k}(M)-b_{k+2}(M), \quad k \geq n+1,
$$

where $\omega$ is the Kähler form.

(ii) Let $M$ be a (non-compact) exact symplectic manifold of dimension $2 n$ and of finite type. Then

$$
c_{k}(M, \omega)=b_{k}(M)+b_{k+1}(M), \quad \text { for } \quad k \geq n+1 .
$$

Proof. $(i)$ follows since $C_{k+2}$ identically vanishes, by using the Lepage's decomposition for harmonic forms. To prove $(i i)$ it is sufficient to notice that $H\left(L_{\omega}\right)$ identically vanishes. 
Since the Betti numbers are topological invariants for compact manifolds, the coeffective cohomology groups are topological invariants for compact Kähler manifolds. Moreover, they measure the jumps of the Betti numbers.

In [15] we have proved, using the Moser Stability Theorem [27, 26, 36], that the coeffective cohomology does not depend on $\omega$, but on the isotopy class of $\omega$. We recall that two symplectic forms $\omega_{0}$ and $\omega_{1}$ are said to be isotopic if they can be joined by a continuous 1-parameter family of cohomologous symplectic structures $\omega_{t}, 0 \leq t \leq 1$. Actually, we can prove something stronger, that is, the coeffective cohomology depends only on the de Rham cohomology class of $\omega$.

Proposition 5.4. Let $(M, \omega)$ be a symplectic manifold of finite type. Then the coeffective cohomology groups $H_{\omega}^{k}(\mathcal{A}(M))$ are invariants of the de Rham cohomology class of $\omega$, for $k \geq n+1$.

Pr o of. We decompose the long exact sequence (2) in 5-term exact sequences,

$$
\begin{aligned}
0 \rightarrow \operatorname{ker} C_{k+1}=\operatorname{Im} H\left(L_{\omega}^{k-1}\right) \stackrel{i}{\rightarrow} H^{k+1}(M) \stackrel{C_{k+1}}{\rightarrow} H_{\omega}^{k}(\mathcal{A}(M)) & \stackrel{H(i)}{\rightarrow} H^{k}(M) \stackrel{H\left(L_{\omega}\right)}{\rightarrow} \operatorname{Im} H\left(L_{\omega}^{k}\right) \rightarrow 0
\end{aligned}
$$

for $k \geq n+1$. Then, we have

$$
c_{k}(M, \omega)=b_{k}(M)+b_{k+1}(M)-\operatorname{dim} \operatorname{Im} H\left(L_{\omega}^{k-1}\right)-\operatorname{dim} \operatorname{Im} H\left(L_{\omega}^{k}\right) .
$$

COROLlary 5.5. If the mapping $L_{\omega}$ is also surjective in cohomology, that is, the mapping $H\left(L_{\omega}\right): H^{k-1}(M) \longrightarrow H^{k+1}(M)$ is surjective for $k \geq n+1$, then

$$
c_{k}(M, \omega)=b_{k}(M)-b_{k+2}(M),
$$

and in such a case, $b_{k}(M) \geq b_{k+2}(M)$.

Corollary 5.6. $c_{k}(M, \omega) \geq b_{k+1}(M)-b_{k-1}(M)$, for $k \geq n+1$.

As is well-known, a compact Kähler manifold is formal [11]. On the other hand, its coeffective numbers are topological invariants and satisfy (4). Moreover, a compact Kähler manifold satisfies the Hard Lefschetz theorem. Lupton and Oprea have suggested [24] the following problem.

Problem 5.7. Let $M$ be a compact symplectic manifold which is formal or satisfies the Hard Lefschetz theorem. Is (4) satisfied then?

The answer to Problem 5.7 in the case of compact symplectic manifolds satisfying the Hard Lefschetz theorem is affirmative. Concretely, for a compact symplectic manifold $\left(M^{2 n}, \omega\right)$ satisfying the Hard Lefschetz theorem, that is,

$$
H\left(L_{\omega}\right)^{r}: H^{n-r}(M) \longrightarrow H^{n+r}(M)
$$

is an isomorphism, we observe that the mapping $H\left(L_{\omega}\right): H^{k-1}(M) \longrightarrow H^{k+1}(M)$ is surjective for $k \geq n+1$, therefore, from Corollary 5.5 the equality (4) is satisfied.

Some evidence for Problem 5.7 (in the formal case) is given by the following results.

i) Complex projective spaces. As we know, $\mathbb{C P}^{n}$ is formal [34]. On the other hand, the cohomology ring of $\mathbb{C P}^{n}$ is $H^{\cdot}\left(\mathbb{C P}^{n}\right)=\mathbb{R}[y] /<y^{n+1}>$, where the degree of $y$ is two. 
Then, for any symplectic structure $\omega$, the mapping $H\left(L_{\omega}\right)$ is always surjective. Therefore, Corollary 5.5 implies (4).

ii) Compact locally symmetric spaces. A compact locally symmetric space is formal $[20,34]$. Moreover, we have the following.

Proposition 5.8. Let $(M, g)$ be a compact Riemannian (locally) symmetric space with harmonic symplectic structure. Then

$$
c_{k}(M, \omega)=b_{k}(M)-b_{k+2}(M) \quad \text { for } \quad k \geq n+1,
$$

therefore, $b_{k}(M) \geq b_{k+2}(M)$.

Proof. Since the wedge product of harmonic forms is harmonic [20], using Hodge theorem and the surjectivity of $L_{\omega}$, we obtain that the mapping $H\left(L_{\omega}\right)$ is surjective, so we conclude by Corollary 5.5.

We propose to study the equality (4) for the following formal spaces:

iii) homogeneous spaces of maximal rank [34],

iv) $H$-spaces (for example, Lie groups) [34].

Also, it is interesting the study of simply connected symplectic manifolds, which is related to the Lupton-Oprea conjecture [25, 34]: any simply connected symplectic manifold is formal.

REMARK 5.9. Bouché [6] has considered the de Rham cohomology groups truncated by the class of the symplectic form $\omega$, that is,

$$
\widetilde{H}^{k}(M)=\left\{a \in H^{k}(M) \mid a \wedge[\omega]=0\right\}=\operatorname{ker} H\left(L_{\omega}\right) .
$$

For a compact Kähler manifold of dimension $2 n$, there exists an isomorphism

$$
H^{k}(\mathcal{A}(M)) \cong \widetilde{H}^{k}(M), \quad k \geq n+1 .
$$

Thus, Bouché [6] proposed the following problem: is the isomorphism (5) satisfied for any compact symplectic manifold? We answered this question in the negative by constructing a counterexample $[1,13]$. A crucial point here was the proof of a Nomizu's type theorem [13] to compute the coeffective cohomology of a compact symplectic nilmanifold at its Lie algebra level.

In relationship with Bouché's problem we have the following result which is a direct consequence of the long exact sequence in cohomology (2).

Proposition 5.10. Let $(M, \omega)$ be a symplectic manifold of dimension $2 n$ and of finite type. Then the following assertions are equivalent:

(i) $H^{k}(\mathcal{A}(M)) \cong \widetilde{H}^{k}(M)$, for $k \geq n+1$;

(ii) $H\left(L_{\omega}\right): H^{k-1}(M) \longrightarrow H^{k+1}(M)$ is surjective for $k \geq n+1$.

Moreover, in such a case, the equality (4) is satisfied.

REMARK 5.11. Although originally the coeffective cohomology was introduced for symplectic structures, it can be also defined for locally conformal symplectic (l.c.s.) structures [35]. Moreover, in both cases, $\left(\operatorname{Im}^{\prime} L_{\omega}, d\right)$ is a differential complex too. The difference 
between both situations is that for l.c.s. structures the short exact sequence (1) is not a short exact sequence of differential complexes. We have proved the following result.

Proposition 5.12. Let $(M, g, J)$ be an almost Hermitian manifold of dimension $2 n$ with Kähler form $\omega$. If $\left(\operatorname{Im} L_{\omega}, d\right)$ is a differential complex, then $M$ is symplectic or l.c.s.

Remark 5.13. For indefinite Kähler manifolds a coeffective Dolbeault cohomology was introduced and discussed in [21].

5.2. Almost contact manifolds. An almost contact metric structure $(\phi, \xi, \eta, g)$ on a $(2 n+1)$-dimensional manifold $M$ is given by a Riemannian metric $g$ and an almost contact structure $(\phi, \xi, \eta)$ such that

$$
g(\phi X, \phi Y)=g(X, Y)-\eta(X) \eta(Y), \quad X, Y \in \mathbb{X}(M) .
$$

The associated fundamental 2 -form $\Phi$ is defined by

$$
\Phi(X, Y)=g(\phi X, Y), \quad X, Y \in \mathfrak{X}(M) .
$$

It follows that $\Phi^{n} \wedge \eta \neq 0$. If $d \Phi=d \eta=0$, it defines an almost cosymplectic structure, and if $\Phi=d \eta$, it defines a contact structure [3].

Let $(\phi, \xi, \eta, g)$ be an almost contact metric structure on a $(2 n+1)$-dimensional manifold $M$. It is called [3, 29]:

i) normal if $[\phi, \phi]+2 d \eta \otimes \xi=0$.

ii) cosymplectic if it is normal and almost cosymplectic,

iii) quasi-Sasakian if it is normal and $\Phi$ is closed,

iv) Sasakian if it is contact and normal.

It was shown in [9] that $L_{\Phi}$ is injective for $k \leq n-1$, and surjective for $k \geq n$. Therefore, if $\Phi$ is closed, we deduce that $\mathcal{A}_{\Phi}^{k}(M)=\{0\}$, for $k \leq n-1$, and then $H_{\Phi}^{k}(\mathcal{A}(M))=\{0\}$, for $k \leq n-1$. Also, $H^{k+2}\left(\operatorname{Im} L_{\Phi}\right)=H^{k+2}(M)$, for $k \geq n+1$.

Proposition 5.14. Let $M$ be a $(2 n+1)$-dimensional almost contact metric manifold with closed fundamental 2-form $\Phi$ and of finite type. Then

(i) the coeffective numbers $c_{k}(M, \Phi)$ are finite dimensional, for $k \geq n+2$;

(ii) the coeffective cohomology groups are invariants of the de Rham cohomology class of $\Phi$, for degrees $k \geq n+2$.

THEOREM 5.15 [16].

(i) For an almost contact manifold of dimension $2 n+1$ with closed fundamental form, say $d \Phi=0$, and of finite type, we have

$$
b_{k}(M)-b_{k+2}(M) \leq c_{k}(M, \Phi) \leq b_{k}(M)+b_{k+1}(M), \quad \text { for } \quad k \geq n+2 .
$$

(ii) If $M$ is a compact cosymplectic manifold, then

$$
c_{k}(M, \Phi)=b_{k}(M)-b_{k+2}(M), \quad \text { for } \quad k \geq n+2 .
$$

(iii) If $M$ is contact, then

$$
c_{k}(M, \Phi)=b_{k}(M)+b_{k+1}(M), \quad \text { for } \quad k \geq n+2 .
$$


(iv) Let $M$ be a (non-compact) exact almost cosymplectic manifold of dimension $2 n+1$. Then

$$
c_{k}(M, \Phi)=b_{k}(M)+b_{k+1}(M), \quad \text { for } \quad k \geq n+2 .
$$

Therefore, the coeffective cohomology groups are topological invariants for compact cosymplectic manifolds. Moreover, since

$$
b_{k}(M) \geq b_{k+2}(M), \quad k \geq n+1,
$$

for compact cosymplectic manifolds [10], then the coeffective numbers measure the jumps of the Betti numbers.

Problem 5.16. Since a compact cosymplectic manifold is formal [9] and satisfies the Hard Lefschetz theorem [9], we can ask again about the relation of the equality (6) to the formality and the Hard Lefschetz theorem.

REMARK 5.17. The relation between the coeffective cohomology and the truncated de Rham cohomology was discussed in [10, 13].

6. Closed 3-forms: calibrated $G_{2}$-manifolds. A $G_{2}$-manifold is a 7-dimensional Riemannian manifold with a 2 -fold vector cross product [7]. Such a manifold $M$ has a nowhere vanishing differential 3-form $\varphi$, which is called the fundamental 3-form of $M$. A $G_{2}$-manifold $M$ is said to be: calibrated if $\varphi$ is closed (i.e. $d \varphi=0$ ), cocalibrated if $\varphi$ is coclosed (i.e. $d * \varphi=0$ ), nearly parallel if $d * \varphi=0$ and $d \varphi=k * \varphi$, where $k \in \mathbb{R}$, and parallel if $\nabla \varphi=0$ (i.e. $d \varphi=0, d * \varphi=0$ ).

Fernández and Gray [12] have proved that $\Lambda^{k}(M)$ splits orthogonally into components $\Lambda_{l}^{k}(M)$ of dimension $l$, which are irreducible under the action of $G_{2}$ :

$$
\Lambda^{1}(M), \quad \Lambda^{2}(M)=\Lambda_{7}^{2}(M) \oplus \Lambda_{14}^{2}(M), \quad \Lambda^{3}(M)=\Lambda_{1}^{3}(M) \oplus \Lambda_{7}^{3}(M) \oplus \Lambda_{27}^{3}(M),
$$

and the others are obtained from these using the Hodge star operator $*$. These spaces are:

$$
\begin{aligned}
\Lambda_{7}^{2}(M) & =\left\{*(\alpha \wedge * \varphi) \mid \alpha \in \Lambda^{1}(M)\right\}, \\
\Lambda_{14}^{2}(M) & =\left\{\beta \in \Lambda^{2}(M) \mid \beta \wedge * \varphi=0\right\}, \\
\Lambda_{1}^{3}(M) & =\left\{f \varphi \mid f \in C^{\infty}(M)\right\}, \\
\Lambda_{7}^{3}(M) & =\left\{*(\alpha \wedge \varphi) \mid \alpha \in \Lambda^{1}(M)\right\}, \\
\Lambda_{27}^{3}(M) & =\left\{\gamma \in \Lambda^{3}(M) \mid \gamma \wedge * \varphi=\gamma \wedge \varphi=0\right\} .
\end{aligned}
$$

For some classes of $G_{2}$-manifolds one can define coeffective complexes (for example, with respect to $\varphi$ and $* \varphi$ ) and other related complexes $[32,31,18,19]$. In this and the following section we will recall some results on these complexes.

Now, let $M$ be a calibrated $G_{2}$-manifold. Then one can consider the coeffective complex with respect to the closed 3 -form $\varphi$. The spaces of $\varphi$-coeffective forms on $M$ are:

$$
\begin{aligned}
& \mathcal{A}_{\varphi}^{i}(M)=\{0\}, \quad i=0,1,2, \\
& \mathcal{A}_{\varphi}^{3}(M)=\Lambda_{1}^{3}(M) \oplus \Lambda_{27}^{3}(M), \\
& \mathcal{A}_{\varphi}^{4}(M)=\Lambda_{7}^{4}(M) \oplus \Lambda_{27}^{4}(M), \\
& \mathcal{A}_{\varphi}^{i}(M)=\Lambda^{i}(M), \quad i=5,6,7 .
\end{aligned}
$$


Also, we can consider the complementary complex $(\mathcal{B}(M), \stackrel{\vee}{d})$, where $\stackrel{\vee}{d}$ is the orthogonal projection of $d$, so that we have the short exact sequence of differential complexes

$$
0 \longrightarrow(\mathcal{A}(M), d) \stackrel{i}{\longrightarrow}(\Lambda(M), d) \stackrel{p}{\longrightarrow}(\mathcal{B}(M), \stackrel{\vee}{d}) \longrightarrow 0
$$

where $i$ and $p$ denote the natural inclusion and the orthogonal projection, respectively. Therefore we have also the long exact sequence in cohomology (for a more complete description see [19]).

We have the following results.

Proposition 6.1 [19].

(i) The coeffective complex $\left(\mathcal{A}_{\varphi}(M), d\right)$ is elliptic in degree $k($ for $k \neq 2)$;

(ii) the complementary complex $(\mathcal{B} \cdot(M), d)$ is elliptic in degree $k($ for $k \neq 3)$;

(iii) in the compact case, the cohomology groups of both complexes are finite dimensional in the ellipticity degrees;

(iv) $c_{i}(M, \varphi)=0(i=0,1,2,3)$ and $c_{i}(M, \varphi)=b_{i}(M)(i=5,6)$;

THEOREM 6.2 [19]. If $M$ is a compact parallel $G_{2}$-manifold, then

$$
c_{4}(M, \varphi)=b_{4}(M)-b_{7}(M), \quad c_{5}(M, \varphi)=b_{5}(M) .
$$

REMARK 6.3. In general, for any $G_{2}$-manifold the sequences $\left(\mathcal{A}_{\varphi}(M), d\right)$ and $(\mathcal{B} \cdot(M), \stackrel{\vee}{d})$ are differential complexes if and only if $M$ is a locally conformal calibrated $G_{2}$-manifold (see [19]).

\section{Closed 4-forms: cocalibrated $G_{2}$ and quaternionic manifolds}

7.1. Cocalibrated $G_{2}$-manifolds. Let $M$ be a cocalibrated $G_{2}$-manifold. Then one can consider the coeffective complex with respect to the 4 -form $* \varphi$. The spaces of $(* \varphi)$ coeffective forms are:

$$
\begin{aligned}
& \mathcal{A}_{* \varphi}^{i}(M)=\{0\}, \quad i=0,1, \\
& \mathcal{A}_{* \varphi}^{2}(M)=\Lambda_{14}^{2}(M), \\
& \mathcal{A}_{* \varphi}^{3}(M)=\Lambda_{7}^{3}(M) \oplus \Lambda_{27}^{3}(M), \\
& \mathcal{A}_{* \varphi}^{i}(M)=\Lambda^{i}(M), \quad i=4,5,6,7 .
\end{aligned}
$$

The coeffective complex is the complementary of the differential complex defined by S. Salamon [32] and whose ellipticity was studied by R. Reyes [31]:

$$
0 \longrightarrow \Lambda^{0}(M) \stackrel{\stackrel{\vee}{D}}{\longrightarrow} \Lambda^{1}(M) \stackrel{\stackrel{\vee}{D}}{\longrightarrow} \Lambda_{7}^{2}(M) \stackrel{\stackrel{\vee}{D}}{\longrightarrow} \Lambda_{1}^{3}(M) \longrightarrow 0
$$

where the differential $\stackrel{\vee}{D}$ is the orthogonal projection of $d$. Again, we can consider the associated exact sequences (for a complete description see [18]).

We have the following results.

Proposition 7.1 [18].

(i) $c_{i}(M, * \varphi)=0,(i=0,1)$ and $c_{i}(M, * \varphi)=b_{i}(M),(i \geq 4)$; 
(ii) if $M$ is a compact nearly-parallel non-parallel $G_{2}$-manifold, then $c_{2}(M, * \varphi)=$ $b_{2}(M)$

(iii) if $M$ is a compact parallel $G_{2}$-manifold we get $c_{2}(M, * \varphi)=b_{2}(M)-b_{1}(M), c_{3}(M, * \varphi)$ $=b_{3}(M)-b_{0}(M) \geq b_{1}(M)$, and then

$$
b_{2}(M) \geq b_{1}(M), \quad b_{3}(M) \geq b_{0}(M)+b_{1}(M) .
$$

REMARK 7.2. The above complexes can even be defined for integrable $G_{2}$-manifolds, that is, locally conformal cocalibrated $G_{2}$-manifolds (see [18]).

7.2. Quaternionic manifolds. A $4 n$-dimensional manifold $M$ is said to be almost quaternionic if there is a subbundle $\mathcal{Q}$ of EndTM such that for each $x \in M$ there is a neighborhood $U$ over which $\mathcal{Q}_{\mid U}$ has a basis $J_{1}, J_{2}, J_{3}$ satisfying

$$
J_{1}^{2}=J_{2}^{2}=-1 \quad \text { and } J_{3}=J_{1} J_{2}=-J_{2} J_{1} .
$$

A Riemannian metric $g$ on $M$ is said to be compatible with $\mathcal{Q}$ if $g(A X, A Y)=g(X, Y)$, for each $X, Y \in T_{x} M$ and $A \in \mathcal{Q}_{x}$ such that $A^{2}=-1$. A compatible metric $g$ can be constructed from any Riemannian metric $g^{\prime}$ by putting

$$
g(X, Y)=\frac{1}{4}\left(g^{\prime}(X, Y)+g^{\prime}\left(J_{1} X, J_{1} Y\right)+g^{\prime}\left(J_{2} X, J_{2} Y\right)+g^{\prime}\left(J_{3} X, J_{3} Y\right)\right)
$$

An almost quaternionic manifold with a compatible metric is called a quaternionic Hermitian manifold.

We may embed $\mathcal{Q}$ isometrically in $\Lambda^{2} T^{*} M$ by mapping an element $A \in \mathcal{Q}$ to the 2 -form $\omega_{A}$ defined by

$$
\omega_{A}(X, Y)=g(X, A Y) \text {, for } X, Y \in T_{x} M .
$$

This enables us to define a global, non-degenerate 4 -form $\Omega$, the fundamental 4 -form $[22,4]$, by

$$
\Omega=\omega_{J_{1}} \wedge \omega_{J_{1}}+\omega_{J_{2}} \wedge \omega_{J_{2}}+\omega_{J_{3}} \wedge \omega_{J_{3}},
$$

where $J_{1}, J_{2}, J_{3}$ is a local basis as above. A quaternionic Hermitian manifold $M$ is said to be:

i) quaternionic almost Kähler if $d \Omega=0$,

ii) quaternionic Kähler if $\nabla \Omega=0$, where $\nabla$ is the Levi-Civita connection.

In dimension 4 both conditions are trivially satisfied. Furthermore, for any dimension $4 n$, the condition $\nabla \Omega=0$ implies the condition $d \Omega=0$, and Swann [33] has proved that the converse also holds for dimension $4 n \geq 12$.

A quaternionic Kähler manifold is said to be hyperkähler if the almost complex structures $J_{1}, J_{2}, J_{3}$ are globally defined and

$$
d \omega_{J_{1}}=d \omega_{J_{2}}=d \omega_{J_{3}}=0 .
$$

Hitchin (see [33]) has shown that this implies that $J_{1}, J_{2}, J_{3}$ are integrable, and hence they define three Kähler structures on $M$. If $M$ is a quaternionic Kähler manifold with zero scalar curvature it can be shown that $M$ is locally hyperkähler.

Let $(M, \mathcal{Q}, g)$ be a quaternionic almost Kähler manifold of dimension $4 n$ with fundamental 4 -form $\Omega$. 
In $[5,17]$ it was shown that the mapping $L_{\Omega}: \Lambda^{k}(M) \longrightarrow \Lambda^{k+4}(M)$ is injective for $k \leq 2 n-2$ and surjective for $k \geq 2 n-2$. Moreover, its adjoint $\Lambda$ with respect to the Riemannian metric $g$ is injective for $k \geq 2 n+2$ and surjective for $k \leq 2 n+2$. As a consequence, we have $H^{k}(\mathcal{A}(M))=\{0\}$, for $k \leq 2 n-2$.

Since $L_{\Omega}\left(\Lambda^{k}(M)\right)=\Lambda^{k+4}(M)$, for $k \geq 2 n-2$ we deduce that

$$
H^{k+4}(\operatorname{Im} L)=H^{k+4}(M),
$$

for $k \geq 2 n-1$. Then proceeding as in the precedent sections we obtain the following result.

Proposition 7.3. Let $M$ be a 4 -dimensional quaternionic almost Kähler manifold of finite type. Then the coeffective numbers $c_{k}(M, \Omega)$ are finite, for $k \geq 2 n$. Also, the coeffective numbers are invariants of the de Rham cohomology class of $\Omega$.

Moreover, we also deduce

THEOREM 7.4.

(i) Let $M$ be a 4 -dimensional quaternionic almost Kähler of finite type. Then

$$
b_{k}(M)-b_{k+4}(M) \leq c_{k}(M, \Omega) \leq b_{k}(M)+b_{k+3}(M), \quad \text { for } \quad k \geq 2 n .
$$

(ii) If $M$ is a compact quaternionic Kähler manifold, then

$$
c_{k}(M, \Omega)=b_{k}(M)-b_{k+4}(M), \quad \text { for } \quad k \geq 2 n .
$$

(iii) If $M$ is a (non-compact) exact almost quaternionic Kähler manifold of finite type, then

$$
c_{k}(M, \Omega)=b_{k}(M)+b_{k+3}(M), \quad \text { for } \quad k \geq 2 n .
$$

Therefore, the coeffective numbers are bounded by upper and lower limits depending on the Betti numbers of the manifold. A first consequence of $(7)$ is that $c_{k}(M, \Omega)=b_{k}(M)$, for $k=4 n-2,4 n-1$ and $4 n$.

REMARK 7.5. The relationship between the coeffective cohomology and the truncated de Rham cohomology by the fundamental 4-form was discussed in [17].

Acknowledgments. This work has been partially supported by grants DGICYT (Spain), Projects PB94-0106, PB-94-0633-C02-02, and by grant UPV, Project 127.310EC 248/96.

\section{References}

[1] L. C. de Andrés, M. Fernández, M. de León, R. Ibáñez and J. Mencía, On the coeffective cohomology of compact symplectic manifolds, C. R. Acad. Sci. Paris, 318, Série I, (1994), 231-236.

[2] M. Berger, Sur les groupes d'holonomie des variétés à connexion affine et des variétés riemannienes, Bull. Soc. Math. France, 83 (1955), 279-330.

[3] D. E. Blair, Contact Manifolds in Riemannian Geometry, Lecture Notes in Math. 509, Springer-Verlag, Berlin, 1976. 
[4] E. Bonan, Sur l'algèbre extérieure d'une variété presque hermitienne quaternionique, C. R. Acad. Sci. Paris, 295, Série I (1982), 115-118.

[5] E. Bonan, Isomorphismes sur une variété presque hermitienne quaternionique, in: Proc. of the Meeting on Quaternionic Structures in Math. and Physics, Trieste, SISSA, (1994), pp. $1-6$.

[6] T. Bouché, La cohomologie coeffective d'une variété symplectique, Bull. Sci. Math., 114 (2) (1990), 115-122.

[7] G. B. Brown and A. Gray, Vector cross products, Comment. Math. Helv. 42 (1967), 222-236.

[8] F. Cantrijn, L. Ibort and M. de León, On the geometry of multisymplectic manifolds, to appear in Journal of the Australian Mathematical Society.

[9] D. Chinea, M. de León and J. C. Marrero, Topology of cosymplectic manifolds, J. Math. Pures et Appl., 72 (6) (1993), 567-591.

[10] D. Chinea, M. de León and J. C. Marrero, Coeffective cohomology on cosymplectic manifolds, Bull. Sci. Math., 119 (1) (1995), 3-20.

[11] P. Deligne, Ph. Griffiths, J. Morgan and D. Sullivan, Real homotopy theory of Kähler manifolds, Invent. Math. 29 (1975), 245-274.

[12] M. Fernández and A. Gray, Riemannian manifolds with structure group $G_{2}$, Ann. Mat. Pura Appl. (IV) 32 (1982), 19-45.

[13] M. Fernández, R. Ibáñez and M. de León, A Nomizu's theorem for the coeffective cohomology, Math. Z. 226 (1997), 11-23.

[14] M. Fernández, R. Ibáñez and M. de León, The coeffective cohomology for compact symplectic nilmanifolds, in: Proceedings of the III Fall Workshop Differential Geometry and its Applications, Granada, Sept. 26-27, 1994, Anales de Física, Monografías 2, CIEMAT-RSFE, Madrid, 1995, pp. 131-144.

[15] M. Fernández, R. Ibáñez and M. de León, Coeffective and de Rham cohomologies of symplectic manifolds, to appear in J. of Geometry and Physics 491 (1998).

[16] M. Fernández, R. Ibáñez and M. de León, Coeffective and de Rham cohomologies on almost contact manifolds, Differential Geometry and Its Applications 8 (1998), 285-303.

[17] M. Fernández, R. Ibáñez and M. de León, Coeffective cohomology of quaternionic Kähler manifolds, Conference on Differential Geometry, Budapest, July 27-30, 1996.

[18] M. Fernández and L. Ugarte, Dolbeault cohomology for $G_{2}$-manifolds, Geometriae Dedicata 70 (1998), 57-86.

[19] M. Fernández and L. Ugarte, A differential complex for locally conformal calibrated $G_{2}$-manifolds, preprint 1996.

[20] Ph. Griffiths and J. Morgan, Rational homotopy theory and differential forms, Progress in Math. 16, Birkhäuser, 1981.

[21] R. Ibáñez, Coeffective-Dolbeault cohomology of compact indefinite Kähler manifolds, Osaka J. Math. 34 (1997), 553-571.

[22] V. Kraines, Topology of quaternionic manifolds, Trans. Amer. Math. Soc. 122 (1966), $357-367$.

[23] P. Libermann and Ch. M. Marle, Symplectic geometry and analytical mechanics, Kluwer, Dordrecht, 1987.

[24] G. Lupton and J. Oprea, private communication.

[25] G. Lupton and J. Oprea, Symplectic manifolds and formality, J. Pure and Appl. Algebra 91 (1994), 193-207. 
[26] D. McDuff and D. Salamon, Introduction to symplectic topology, Oxford Math. Monographs, Oxford Univ. Press, 1995.

[27] J. Moser, On the volume elements on manifolds, Trans. Amer. Soc. Math., 120 (1965), $286-295$.

[28] K. Nomizu, On the cohomology of compact homogeneous spaces of nilpotent Lie group, Annals of Math. 59 (2) (1954), 531-538.

[29] J. A. Oubiña, New classes of almost contact metric structures, Publicationes Mathematicae 32 (3-4) (1985), 187-193.

[30] M. S. Raghunatan, Discrete subgroups of Lie groups, Ergebnisse der Mathematik 68, Springer-Verlag, Berlin, 1972.

[31] R. Reyes, Some special geometries defined by Lie groups, Thesis, Oxford, 1993.

[32] S. Salamon, Riemannian Geometry and Holonomy Groups, Pitman Research Notes in Math. Series 201, Longman, Boston, 1989.

[33] A. Swann, Hyperkähler and quaternionic Kähler geometry, Math. Ann. 289 (1991), 421450 .

[34] A. Tralle and J. Oprea, Symplectic Manifolds with no Kähler Structure, Lecture Notes in Math. 1661, Springer, Berlin, 1997.

[35] I. Vaisman, Locally conformal symplectic manifolds, Internat. J. Math. \& Math. Sci. 8 (1985), 3, 521-536.

[36] A. Weinstein, Lectures on symplectic manifolds, CBMS, Amer. Math. Soc. 29, Providence (R.I.), 1977. 